Service social

\title{
Violence conjugale et paternité : les défis de l'intervention sociale
}

\author{
Chantal Bourassa, Michel Labarre, Pierre Turcotte, Geneviève Lessard et \\ Nicole Letourneau
}

Volume 60, numéro 1, 2014

Des enfants, des couples et des familles.

URI : https://id.erudit.org/iderudit/1025134ar

DOI : https://doi.org/10.7202/1025134ar

Aller au sommaire du numéro

\section{Éditeur(s)}

École de service social de l’Université Laval

ISSN

1708-1734 (numérique)

Découvrir la revue

Citer cet article

Bourassa, C., Labarre, M., Turcotte, P., Lessard, G. \& Letourneau, N. (2014).

Violence conjugale et paternité : les défis de l'intervention sociale. Service

social, 60(1), 72-89. https://doi.org/10.7202/1025134ar
Résumé de l'article

Cet article présente les résultats d'une recherche qualitative visant à comprendre comment les intervenants intègrent le concept de paternité dans les groupes de thérapie pour les hommes aux comportements violents. Des groupes de discussion et des entrevues semi-dirigées ont été effectués auprès d'intervenants dans trois organismes au Nouveau-Brunswick et au Québec. Selon l'analyse des données recueillies, la prise en compte de la paternité des hommes représente un défi pour ces intervenants. Les difficultés qu'éprouvent de nombreux hommes (non-reconnaissance de leur violence, expériences de violence lors de l'enfance, frustrations en lien avec les systèmes et problèmes de toxicomanie ou de santé mentale), le manque de temps, d'outils et de formation pour les intervenants, les questions de sécurité, et les normes culturelles et représentations sociales de la violence conjugale, compliquent l'exploration de la paternité dans le cadre des groupes d'intervention. 


\title{
Violence conjugale et paternité : les défis de 1'intervention sociale ${ }^{1}$
}

\author{
BOURASSA, Chantal \\ LABARRE, Michel \\ TURCOTTE, Pierre \\ LESSARD, Geneviève \\ LETOURNEAU, Nicole
}

\section{RÉSUMÉ}

Cet article présente les résultats d'une recherche qualitative visant à comprendre comment les intervenants intègrent le concept de paternité dans les groupes de thérapie pour les hommes aux comportements violents. Des groupes de discussion et des entrevues semi-dirigées ont été effectués auprès d'intervenants dans trois organismes au Nouveau-Brunswick et au Québec. Selon l'analyse des données recueillies, la prise en compte de la paternité des hommes représente un défi pour ces intervenants. Les difficultés qu'éprouvent de nombreux hommes (non-reconnaissance de leur violence, expériences de violence lors de l'enfance, frustrations en lien avec les systèmes et problèmes de toxicomanie ou de santé mentale), le manque de temps, d'outils et de formation pour les intervenants, les questions de sécurité, et les normes culturelles et représentations sociales de la violence conjugale, compliquent l'exploration de la paternité dans le cadre des groupes d'intervention.

Mots-clés : Violence conjugale, paternité, hommes aux comportements violents, pratique des intervenants.

\begin{abstract}
This article reports the results of a qualitative research aimed at understanding how facilitators of male domestic violence groups consider fathering in their interventions with these men. Group discussions and interviews were conducted with facilitators working in three organisations in Quebec and New Brunswick. According to those facilitators, it is difficult to integrate fathering in their interventions. The numerous difficulties experienced by the men (non-recognition of their violence, violence experienced during childhood, frustrations toward the systems, and mental health or addiction problems), lack of time, tools and education and questions regarding security, complicate exploration of fathering in these groups.
\end{abstract}

Key-words: Domestic violence, fatherhood, men who are violent, professional practices.

1 Recherche subventionnée par le CRSH (2010-2013). 


\section{INTRODUCTION}

La paternité et la violence conjugale (VC) sont deux constructions sociales qui ont, jusqu'à récemment, été envisagées dans des univers conceptuels distincts, et ce, tant sur le plan de la recherche que sur celui de l'intervention. Historiquement, la violence des hommes à l'égard des femmes a été tolérée, alors que le rôle de père était limité à celui de maître de la discipline et de pourvoyeur pour la famille. Grâce, d'une part, au mouvement social d'émancipation des femmes, la VC a été reconnue socialement comme un problème grave nécessitant des mesures de protection efficaces pour les femmes victimes et de punition pour les hommes violents (Turcotte, 1997). D'autre part, le mouvement en faveur de l'engagement paternel a permis de valoriser le lien émotif entre le père et l'enfant (Deslauriers, 2008 ; Schoppe-Sullivan et al., 2004). Ces deux mouvements soutiennent des intérêts qui peuvent diverger, soit la protection des personnes victimes et la promotion de l'engagement paternel, ce qui occasionne des débats et des contradictions dans les pratiques (Cabrera et Peters, 2000 ; Edleson et Williams, 2007).

La récente attention portée à la paternité dans un contexte de VC suscite plusieurs controverses (Harne, 2011 ; Holden et Baker, 2004 ; Lessard et al., 2010). Certains auteurs soutiennent que les hommes aux comportements violents manipulent le système juridique à leur avantage et utilisent les enfants dans le but de contrôler la mère ; ainsi, les contacts entre eux et leurs enfants ne sont pas à encourager (Bancroft et al., 2012; MacDonald et al., 2009). D'autres estiment que la paternité de ces hommes doit faire l'objet d'intervention, puisque ceux-ci ont des contacts réguliers avec leurs enfants, même s'ils sont reconnus coupables d'avoir commis des actes de VC (Deslauriers, 2008 ; Salisbury et al., 2009) ou s'il y a séparation conjugale (Peled et Perel, 2007 ; Scott et al., 2007). À cet égard, certains laissent à penser qu'il y a des hommes, en processus de changement, qui désirent établir une bonne relation avec leur enfant ; ainsi leur paternité devrait être abordée en intervention (Bourassa et al., 2013 ; Perel et Peled, 2008). D'ailleurs, des auteurs croient qu'une intervention sur la paternité peut avoir des effets bénéfiques sur l'enfant et sa relation avec son père (Fleck-Henderson et Areán, 2004 ; Mandel, 2002 ; Neugut et al., 2010 ; Peled et Perel, 2007 ; Stover et al., 2011). En fait, des études indiquent que les pères qui tentent de reconstruire une relation positive et sans violence avec leurs enfants réduisent le risque de transmission intergénérationnelle de la violence (FleckHenderson et Areán, 2004 ; Scott et al., 2007). De plus, les pères semblent avoir plus de facilité à reconnaître les conséquences négatives de leur violence sur leurs enfants que sur leur conjointe, et ils sont plus enclins à se responsabiliser lorsqu'ils réalisent les dommages causés à leur enfant ; en ce sens, une réflexion sur leur relation avec leur enfant constitue un catalyseur de changement (Neugut et al., 2010).

Par ailleurs, on connaît très peu le point de vue des intervenants sociaux concernant la paternité en contexte de VC et la façon dont ils font face à ces nombreuses controverses au sein de leur pratique. Cet article vise à présenter une étude canadienne effectuée auprès d'intervenants animant des groupes d'intervention pour hommes ayant des comportements violents, afin de comprendre comment ils abordent la paternité. Pour débuter, nous présentons les écrits traitant des interventions sur la paternité. Par la suite, nous exposons le cadre théorique puis la méthodologie de l'étude. Après avoir présenté les résultats des données 
recueillies et la discussion, nous aborderons, en conclusion, les retombées pour la pratique et la recherche.

\section{Recension des écrits}

Pour cette recension des écrits, il est d'abord question des résultats des recherches qui se sont penchées sur la façon dont les organismes offrant des services axés sur la paternité considèrent la VC. Ensuite, nous présentons la seule étude répertoriée qui ait porté sur la paternité dans le cadre des groupes d'intervention en VC. Cette partie se termine par la mention d'initiatives qui visent spécifiquement la paternité en contexte de VC.

Les quelques études réalisées auprès d'intervenants œuvrant dans les organismes d'aide aux pères indiquent que la VC est rarement prise en compte lors de l'intervention (Cronholm et al., 2011 ; Roulet, 2003 ; Williams et al., 2001). En fait, ces intervenants se questionnent sur la façon dont ils peuvent prendre en considération la VC, particulièrement si les programmes qu'ils offrent soutiennent activement l'engagement du père à l'égard de ses enfants et des autres membres de famille (Roulet, 2003). Certains intervenants préfèrent ne pas aborder la VC, ni la dépister, craignant qu'une intervention inadéquate ou inefficace puisse avoir des effets néfastes sur les programmes pour les pères (Roulet, 2003). D'autres intervenants expliquent cette situation par le manque de formation et de connaissances en VC, de même que par la croyance que le dépistage de la violence ne fait pas partie du mandat de leur organisme (Cronholm et al., 2011).

Du côté des organismes en VC, les objectifs de leurs programmes misent sur l'arrêt des comportements violents des hommes et non sur la paternité (Edleson et Williams, 2007 ; Guille, 2004 ; Peled et Perel 2007). Dès leur début, les programmes ont été encadrés par l'approche féministe psycho-éducative, définissant la VC comme une question de pouvoir et de contrôle des hommes sur les femmes, ce qui a favorisé la mise en place d'interventions selon une logique de contrôle social visant à rendre les hommes responsables de leur violence (AugustaScott, 2009 ; Turcotte, 1997). Les hommes ont été réduits à leurs comportements violents, de telle sorte que les autres aspects de leur identité ont reçu peu d'attention, notamment la dimension parentale.

Nous n'avons répertorié qu'une seule étude, effectuée en Suède, qui se soit penchée spécifiquement sur la façon dont la paternité est prise en compte par des intervenants œuvrant dans des organismes pour hommes ayant des comportements violents (Edin et al., 2009). Selon les six intervenants interviewés, il apparaît ardu d'intégrer la paternité dans le cadre de leurs interventions ; les pères, contrairement aux mères, n'abordent pas spontanément des sujets ayant trait à leurs enfants. Également, selon ces intervenants, le mandat des programmes qui portent sur la cessation des comportements violents, le manque de temps et le nombre limité de rencontres créent des obstacles à l'intervention sur la paternité. Les auteurs de l'étude ont aussi mentionné qu'il se peut que les intervenants évitent d'aborder la paternité, de peur que les pères ne dévoilent des informations laissant sous-entendre que la sécurité et le bien-être d'un enfant sont compromis, ce qui les obligerait à signaler la situation aux services de protection de l'enfance. 
En dépit de ces lacunes sur le plan des connaissances, certaines initiatives récentes s'intéressent précisément à l'intervention sur la paternité en contexte de VC. Le programme Caring Dads a été développé à London en Ontario (Scott et Crooks, 2006) et vise les pères ayant des comportements de maltraitance à l'égard de leur enfant, l'exposition à la VC étant considérée comme une forme de maltraitance. II s'agit d'un programme de 17 rencontres ; une évaluation récente révèle que le programme a des effets positifs sur les pères, notamment en ce qui a trait à la façon de réagir face aux comportements difficiles de leur enfant et à la façon de percevoir l'autre parent (plus de respect et moins de jugements) (Scott et Lishak, 2012). D'autre part, l'initiative Fathering After Violence (Areán et Davis, 2007 ; Fleck-Henderson et Areán, 2004) a été conçue à Boston et englobe trois activités qui peuvent être intégrées aux programmes d'intervention destinés aux hommes ayant des comportements violents ou autres programmes pour les pères. Outre ces initiatives, quelques auteurs ont également contribué aux connaissances sur l'intervention auprès des pères ayant des comportements violents en proposant des programmes d'activités à réaliser (Mandel, 2002 ; Peled et Perel, 2007 ; Stover, 2013).

Les auteurs de ces programmes s'entendent sur le fait que la sécurité de la mère et des enfants doit être priorisée lors de l'intervention auprès des pères (Areán et Davis, 2007; Fleck-Henderson et Areán, 2004 ; Scott et Crooks, 2006). Également, la participation des pères doit avoir le potentiel d'améliorer le bien-être des enfants (Fleck-Henderson et Areán, 2004 ; Scott et al., 2007). Certains hommes, en dépit de l'intervention qu'ils reçoivent, continuent d'être violents et, dans ces cas, les contacts avec les enfants peuvent se révéler non souhaitables (Areán et Davies, 2007 ; Fleck-Henderson et Areán, 2004). Une étroite collaboration avec les autres organismes dispensant des services à la famille est donc nécessaire (Scott et al., 2007).

Ces quelques initiatives visant à aborder la paternité en contexte de VC n'ont eu lieu que dans une province canadienne, soit l'Ontario. De plus, aucune étude canadienne, plus spécifiquement au Québec et au Nouveau-Brunswick, n'a porté sur le point de vue des intervenants sur leur façon d'aborder la paternité lorsqu'ils interviennent auprès des hommes aux comportements violents. Afin de pallier ce manque de connaissances, nous avons effectué une recherche auprès d'intervenants œuvrant dans deux provinces canadiennes, dans le but de comprendre comment ils intègrent la paternité dans le cadre des interventions de groupe en VC.

\section{Cadre théorique : l'approche constructiviste des problèmes sociaux}

Selon une approche constructiviste, les mouvements sociaux et les groupes identitaires jouent un rôle très actif dans la construction des problèmes sociaux, engendrant ainsi des luttes de pouvoirs entre les groupes détenant des visions différentes (Mayer, 2001 : 128). L'étude faisant l'objet de cet article porte sur la paternité en contexte de VC, soit une problématique sociale qui suscite de nombreux débats dans la pratique, notamment entre le mouvement social d'émancipation des femmes, visant la protection des femmes victimes, et le mouvement en faveur de l'engagement paternel, visant la promotion du lien émotif entre le père et son enfant (Cabrera et Peters, 2000 ; Edleson et Williams, 2007 ; Deslauriers, 2008). 
Ainsi, pour comprendre les actions des intervenants sur la paternité en contexte de VC, les contextes social, culturel et organisationnel (mandant de l'organisme et approches privilégiées) qui entourent la pratique doivent être pris en compte. Comme l'indique Mayer (2001: 113) : «(...) les activités présumément subjectives et individuelles d'une part, et celles qui sont supposées objectives et sociétales d'autre part, se construisent ensemble dans les divers environnements sociaux, qu'ils soient intimes, institutionnels ou collectifs. » Dans ce sens, adopter une approche constructiviste en recherche nécessite « des descriptions approfondies sur les relations entre les individus et les événements, le langage, la perception et le contexte institutionnel et culturel » (Mayer, 2001 : 113). Dans cette étude, l'analyse du discours sur la façon dont les intervenants interviennent sur la paternité et les éléments qui influent sur ces interventions permettront de cerner comment ils reconstruisent cette paternité en contexte de VC.

\section{MÉTHODOLOGIE}

Cette étude, se situant dans un courant constructiviste, est de nature qualitative. Pour le recrutement, trois organismes offrant des groupes d'aide aux hommes ayant des comportements violents, soit deux du Nouveau-Brunswick et un du Québec, ont participé à l'étude. D'abord, les directions des organismes ont accepté que les intervenants des groupes pour hommes soient contactés par les chercheurs de l'étude afin de solliciter leur participation à la recherche. De ces intervenants potentiels, six intervenants ont refusé de participer en raison d'un manque de temps. En tout, 11 intervenants, québécois $(n=5)$ et néo-brunswickois $(n=6)$, soit 4 femmes et 7 hommes, ont volontairement accepté de participer à l'étude. La plupart des intervenants étaient âgés de 30 à 40 ans, bien que deux appartenaient à la catégorie d'âge 61 ans et plus. Presque tous les participants détenaient un baccalauréat dans une discipline des sciences sociales (sauf un qui détenait un diplôme collégial en technique de travail social et un autre qui détenait une maîtrise en service social). La plupart des intervenants rencontrés (82 \% ou 9 sur 11) avaient des enfants.

La collecte des données s'est effectuée de septembre à décembre 2010. Deux groupes de discussion, un par province, d'une durée d'environ deux heures, comptant respectivement quatre (Québec) et trois (Nouveau-Brunswick) intervenants, ont été constitués. Nous avons rencontré quatre autres participants, ne pouvant être présents lors des groupes de discussion, en entrevues individuelles; ces rencontres ont duré environ une heure. Les entrevues de groupe et les entrevues individuelles ont été animées par un chercheur de l'étude et un assistant de recherche ayant une formation en VC. Le même canevas d'entrevue, à questions ouvertes et semi-dirigées, a été utilisé pour les groupes de discussion et les entrevues individuelles. Ce guide était divisé en deux grandes parties: la première regroupait des questions sur la perception de la paternité en contexte de violence et la deuxième englobait des questions sur l'intervention. Pour cet article, nous présentons les résultats recueillis dans la deuxième partie de l'entrevue traitant plus spécifiquement de la façon dont les intervenant intègrent la paternité dans leur pratique (approches, objectifs et moyens d'intervention) et des défis auxquels ils font face lors des rencontres de groupe. 
Les entrevues ont été enregistrées sur bande audio et transcrites intégralement. Nous avons effectué une analyse thématique à l'aide du logiciel Nvivo (version 9) (Paillé et Mucchelli, 2012). De façon indépendante, l'un des chercheurs de l'étude et l'assistant de recherche ont suivi une démarche de thématisation en continu, identifiant les thèmes pertinents pour ensuite construire de façon simultanée un relevé de thème transversal. Chacun de ces relevés a été comparé et, suite à une entente commune, un arbre thématique a été créé. Afin d'assurer la validité des analyses, cet arbre thématique a été présenté aux autres membres de l'équipe de recherche, afin de susciter une discussion et d'obtenir leurs réactions. De plus, nous avons communiqué les résultats aux intervenants qui ont participé à l'étude afin de solliciter leurs commentaires (Mukamurera et al., 2006). Comme l'indiquent Huberman et Miles (1991 : 442), « les personnes interviewées ou observées constituent une des sources les plus logiques de corroboration ».

\section{RÉSULTATS}

Dans le cadre de cette partie, nous présentons les résultats de l'étude. Dans un premier temps, les objectifs d'intervention en lien avec la paternité des hommes aux comportements violents sont explicités. Par la suite, il sera question des défis rencontrés par les intervenants dans leur pratique lorsqu'ils veulent aborder la paternité dans leurs interventions.

\section{Les objectifs d'intervention en lien avec la paternité des hommes aux comportements violents}

Les intervenants ont mentionné qu'ils combinent plus d'une approche d'intervention dans leur pratique auprès des hommes aux comportements violents: ils s'inspirent à la fois des approches qui ont été traditionnellement utilisées dans les groupes pour hommes aux comportements violents, telles que le modèle cognitif-comportemental et l'approche proféministe psycho-éducationnelle (Babcock et al., 2004), et des approches postmodernes qui conçoivent l'expérience unique et holistique de la personne, de même que la diversité et la complexité des expériences de vie. À cet égard, les intervenants ont évoqué l'approche centrée sur la solution (De Shazer, 1999) et l'approche humaniste-existentielle (Lecomte et Richard, 1999). Les intervenants du Nouveau-Brunswick ont mentionné l'approche narrative (White, 1991), celle-ci encadrant d'ailleurs depuis 2009 le programme d'intervention pour hommes aux comportements violents mis en place par la province (Blanc-Sahnoun et Dameron, 2009).

De ces approches théoriques découlent cinq objectifs d'intervention que les intervenants poursuivent en ce qui a trait particulièrement à la paternité des hommes qu'ils accompagnent : 1) responsabiliser les pères et leur faire prendre conscience des conséquences de leurs comportements violents sur leur enfant ; 2) co-construire une identité de père plus positive ; 3) valoriser le rôle du père ; 4) éduquer les pères aux besoins de leurs enfants et aux façons positives de les discipliner; et 5) renvoyer les pères vers d'autres organismes selon leurs besoins spécifiques. Dans le cadre du premier objectif, les intervenants veulent que les pères prennent conscience des conséquences pour les enfants de la dynamique de violence et du type de modèle qu'ils transmettent à leurs enfants. Ils utilisent la projection de courts métrages démontrant des enfants impliqués dans une scène de VC et entament par la suite avec les pères un échange sur le contenu de ces projections. Ils peuvent aussi explorer le vécu des pères lors de leur enfance, afin d'établir un parallèle entre les effets de la violence qu'ils ont eux- 
mêmes subie et les conséquences de leur propre violence sur leur enfant. Les intervenants veulent que les pères adoptent des comportements qui sont davantage en lien avec les valeurs qu'ils désirent transmettre à leur enfant. Comme le souligne un intervenant, bien que les pères accordent de l'importance à la transmission de valeurs positives à leur enfant, leurs comportements ne sont pas toujours cohérents avec ces valeurs.

Quelle sorte de modèle que tu veux donner à tes enfants ? Modèle de père et tout ça. Quelle sorte d'image qu'il veut donner à ses enfants ? C'est un exercice qu'on fait avec eux autres, on va prendre un flip chart, puis on va marquer toutes sortes d'images qu'eux autres veulent donner à leurs enfants. On travaille sur les pensées et valeurs.

Comme deuxième objectif, les intervenants considèrent important de co-construire avec les pères une meilleure image d'eux-mêmes en misant sur leurs forces car, selon eux, les pères qu'ils accompagnent ont tendance à se percevoir comme de « mauvaises personnes » et de « mauvais pères ». Les intervenants soulignent l'importance d'établir un lien de confiance avec les pères dans une atmosphère de respect et de non-jugement.

Plusieurs hommes viennent au programme et se perçoivent comme de «mauvaises personnes », de « mauvais conjoints », de «mauvais pères », et ils croient que tout le monde dans la société les voit ainsi. Avec l'approche narrative, nous ne mettons pas ces genres d'étiquettes. Ceci fait partie de notre programme de les aider à se percevoir d'une meilleure façon : un bon conjoint, un bon père, un bon partenaire.

Quant au troisième objectif, les intervenants veulent que les pères prennent conscience de leur importance aux yeux de leur enfant. Ils encouragent les pères à passer du temps avec leur enfant et à jouer avec lui. Selon eux, lorsque les pères constatent qu'ils ont un rôle fondamental à jouer auprès de leur enfant, outrepassant le seul fait de contribuer financièrement aux besoins de la famille, ils sont davantage motivés à entreprendre un processus de changement.

On en profite pour aller faire le tour et on se rend compte que ce n'est pas par mauvaise volonté, mais que beaucoup de gars se sentent comme pas capables ou ils n'ont pas confiance qu'ils vont être capables de jouer avec leurs enfants. Ça c'est un, et l'autre c'est : « je n'aime pas les jeux auxquels y jouent. » Alors ça confronte à dire : «toi, tu es le père, est-ce que c'est possible que, un moment donné, tu piles sur ton orgueil pis que tu joues à quelque chose que tu n'aimes pas parce que c'est ton enfant que t'aimes ? " Alors, là, ça aussi, c'est vraiment quelque chose que je trouve d'important parce que souvent ils reviennent pis ils disent : « oui, je l'ai fait, j'ai joué, ç'a marché, j'ai l'impression qu'il y a eu quelque chose qui s'est passé. »

Le quatrième objectif poursuivi par les intervenants est d'éduquer les pères en ce qui a trait aux besoins de leurs enfants et aux façons positives d'exercer la discipline. À cet égard, les intervenants rencontrés qui sont également parents utilisent leur vécu personnel afin de servir de modèles auxquels les pères peuvent se référer.

On est des parents et comme eux, on vit des situations avec nos enfants qu'on doit gérer et qui ne sont pas toujours évidentes comme tous les parents. Ça, je pense que c'est quelque chose qui aide [les pères] à s'ouvrir là-dessus parce qu'il y a beaucoup de peur d'être jugé quand il est question des enfants et de comment ils réagissent avec les enfants. Fait que 
c'est un outil que d'utiliser notre expérience de parent dans le sens d'universaliser ce qu'ils peuvent vivre.

Enfin, étant donné que les groupes d'intervention sont prioritairement, voire exclusivement, axés sur la cessation des comportements violents, les intervenants se doivent de diriger les pères vers d'autres organismes. Il peut s'agir, entre autres, de programmes axés sur le développement d'habiletés parentales, de lignes d'écoute, etc.

En bref, les objectifs en lien avec la paternité misent non seulement sur la responsabilisation et la conscientisation de la violence, mais également sur des éléments positifs, soit d'amener les pères à percevoir leur paternité d'un œil plus positif et à se sentir un parent plus compétent.

\section{Les défis rattachés à l'intervention sur la paternité}

Les intervenants rencontrés ont indiqué qu'il apparaît difficile d'aborder la paternité auprès des hommes ayant des comportements violents, et ce, en raison d'enjeux se situant à trois niveaux, soit individuel (liés au père), organisationnel et social.

\section{Défis en lien avec les pères}

En ce qui a trait aux défis rattachés aux pères, les intervenants ont mentionné que des croyances endossées par certains pères ou des réalités vécues par ceux-ci peuvent freiner le travail sur la paternité, par exemple la non-reconnaissance de leur violence ou de ses effets, les expériences vécues dans l'enfance, la préoccupation face aux conflits avec la mère de l'enfant, les frustrations avec les systèmes juridique et de protection de l'enfance et les problèmes connexes à la violence (toxicomanie, problèmes de santé mentale, etc.).

Les intervenants considèrent qu'ils font face à un défi considérable lorsque les pères nient leur violence et ses conséquences sur l'enfant. Certains pères, bien qu'ils reconnaissent leur violence, minimisent les effets de certaines formes de violence sur leur enfant, en particulier la violence psychologique ou verbale. Ainsi, il se révèle difficile pour les intervenants de prendre en compte la paternité si les pères ne reconnaissent pas la dynamique de la violence et de ses effets sur la relation père-enfant. De la même façon, les intervenants rencontrés mentionnent que la plupart des pères auprès desquels ils interviennent ne sont pas volontaires pour entreprendre un processus de changement. Un grand nombre d'entre eux initient une démarche sous la contrainte d'un tiers en position d'autorité (par exemple, ordonnance de la cours, exigence de l'agence de la protection de l'enfance). Il se peut également que certains hommes décident d'entreprendre une démarche suite à un ultimatum de la conjointe. Ces hommes sont susceptibles d'être peu motivés et de moins s'investir dans leur démarche, le travail sur la paternité apparaît alors plus difficile.

Un autre défi a trait à l'enfance vécue par les pères et le modèle parental auquel ils ont été exposés. Selon les intervenants, la plupart des hommes faisant partie des groupes d'intervention ont été exposés à un modèle de père violent ou absent, ce qui explique qu'ils aient de la difficulté à concevoir l'image d'un « bon père », car ils n'ont pas de point de repère positif. 
De plus, les intervenants ont fait remarquer que certains pères sont préoccupés par les conflits qu'ils vivent avec la mère des enfants ou obsédés par leur rancœur à l'égard des systèmes juridique et de protection de l'enfance. Ces pères réussissent difficilement à se concentrer sur les thèmes discutés dans le groupe. Lorsque les conflits avec la mère des enfants ou les frustrations des pères sont abordés, les intervenants se sentent souvent en terrain glissant car ils considèrent que la ligne est mince entre, d'une part, se montrer compréhensifs face aux injustices que peuvent vivre certains pères et leur offrir un espace pour ventiler et, d'autre part, le risque de déresponsabiliser les hommes et de nourrir les sentiments d'iniquité et de colère qui les habitent.

C'est une limite où on n'ose pas trop rentrer là-dedans parce que ça peut être borderline. Ça peut peut-être les déresponsabiliser plus ou ça peut les alimenter encore plus sur l'injustice qu'ils vivent. Fait que là, on est comme sur une ligne étroite, qui peut déraper parce qu'on a beaucoup de pères qui dérapent beaucoup sur la DPJ [Direction de la protection de la jeunesse]. Fait qu'on ose par trop rentrer là-dedans.

Enfin, les problèmes souvent concomitants à la VC, tels que la toxicomanie, la criminalité et les problèmes de santé mentale, posent des défis supplémentaires pour les intervenants. Étant donné la complexité des situations vécues par certains pères, il leur est malaisé de se concentrer sur leurs enfants et leur rôle de père. Ainsi, dans ces cas, d'autres problèmes doivent être abordés avant de s'intéresser à la paternité.

\section{Défis organisationnels}

Les intervenants ont également mentionné qu'il leur est difficile d'aborder la paternité en raison des limites inhérentes à la composition, à la durée et au mandat des programmes. Puisqu'il n'y a pas de groupes spécifiques pour les pères, il peut être ardu de maintenir l'intérêt du groupe Iorsque seulement quelques hommes sont pères. Ainsi, l'intégration de thèmes rattachés à la paternité varie en fonction du nombre de pères qui se retrouvent dans le groupe. Également, pour les intervenants, le mandat et la durée du programme constituent deux contraintes considérables : le mandat, qui est d'intervenir sur les comportements violents et les attitudes des hommes à l'égard de leur partenaire, exige que certains thèmes soient abordés en premier, ce qui laisse peu de temps à l'inclusion d'autres aspects, tels que la paternité.

Les intervenants ont identifié trois défis rattachés à leur pratique, à savoir leur isolement professionnel, le manque de formation et le manque d'outils appropriés. Les intervenants ont mentionné que leur isolement professionnel constitue un obstacle, car ils n'ont souvent qu'un seul point de vue de la situation, soit celui du père. Par conséquent, il leur semble difficile de juger de l'exactitude des renseignements fournis par les hommes et d'évaluer le degré de dangerosité qu'ils représentent pour leur femme et leurs enfants. Ils déplorent le fait qu'ils aient peu de possibilités d'échange avec les autres professionnels impliqués dans la situation familiale.

Notre intervention puis le sens qu'on va y donner reposent sur une version. Des fois, ça pose des défis parce qu'on n'a pas l'enfant, on n'a pas le regard sur l'environnement familial. 
Des fois, c'est difficile même de se positionner par rapport aux participants, même dans notre tête, à savoir : c'est-tu dangereux comme milieu ?

Les intervenants ont aussi indiqué qu'ils ne disposaient pas d'outils d'intervention et n'avaient pas de formation spécifique sur la paternité des hommes aux prises avec des comportements violents. Cela fait en sorte qu'ils trouvent difficile d'intégrer des thèmes touchant la paternité dans les groupes et de composer avec certaines situations qui se présentent dans leur pratique.

\section{Défis en lien avec le milieu social}

Quelques défis en lien avec le milieu social ont été mentionnés par les intervenants. De prime abord, les lois provinciales concernant la protection des enfants ont été rapportées comme créant des dilemmes au sein de leur pratique en raison du fait que l'exposition à la VC peut être un motif de signalement. Au Nouveau-Brunswick, depuis plusieurs années, l'exposition à la VC est clairement mentionnée dans la Loi sur les services à la famille comme une forme de mauvais traitement pouvant faire l'objet d'un signalement. Au Québec, les mauvais traitements psychologiques sont le nouveau motif de signalement inclus dans la Loi sur la protection de la jeunesse depuis 2006 et, dans cette Loi, l'exposition à la violence familiale est mentionnée parmi les formes de mauvais traitements psychologiques susceptibles de faire l'objet d'un signalement. Les intervenants rencontrés priorisent la sécurité et le bien-être des enfants, mais ils vivent un dilemme dans ce sens car, en faisant un signalement, ils risquent de fragiliser leur relation avec le père.

C'est dur quand tu vois du monde devant toi puis tu ne sais pas ce qui se passe à la maison pis tu te dis: si moi j'appelle, pis je fais un signalement, je perds le lien, c'est terminé bonsoir, merci. C'est quoi l'enjeu le plus important, est-ce que je vais gager sur le fait que ce monsieur-là va faire un bout de chemin pis va s'en sortir pis que les enfants vont être gagnants ? Ou si j'arrête ça tout de suite puis que, là, la rage va recommencer et la guerre va continuer. En tout cas, ce n'est pas évident à porter.

D'autres défis qui ont été présentés par les intervenants concernent plutôt les valeurs et normes de la société et la culture dans lesquelles baignent les hommes. Pour eux, la socialisation masculine constitue une barrière à l'intervention sur la paternité, car elle fait en sorte que les hommes ne soient pas d'emblée portés à discuter de leur relation avec leurs enfants et de leur souffrance intérieure lorsqu'ils se retrouvent entre eux. Lorsqu'ils ressentent de la culpabilité face au fait d'exposer leur enfant à la violence, ils ont tendance à éviter le sujet.

On dirait que ça va être encore plus tabou de parler de « je n'ai pas été un bon père » que de rapporter des éléments de violence qui sont arrivés dans la semaine, sur les manques, sur l'aide qu'ils n'ont pas apportée à la mère.

De plus, la culture d'origine du père peut constituer un obstacle supplémentaire, surtout lorsque les normes et valeurs en ce qui a trait à la $\mathrm{VC}$ et la relation entre le père et son enfant divergent de celles de l'intervenant. Le père peut alors percevoir sa façon d'agir auprès de sa conjointe et de ses enfants comme tout à fait acceptable, alors que ces agissements sont considérés comme de la violence selon les lois canadiennes. II apparaît ainsi difficile pour 
l'intervenant de rejoindre le père afin de lui faire prendre conscience de sa violence et de ses effets nocifs pour l'enfant.

Un autre défi concerne les représentations sociales de la VC et des hommes aux comportements violents. Les services se sont d'abord développés en ayant comme priorité la protection des femmes victimes et la condamnation des hommes violents. Ceux-ci se sont vus étiquetés «hommes violents », de telle sorte que les autres facettes de leur identité ont été voilées ; par conséquent, ces hommes ont reçu peu de soutien pour exercer leur paternité.

Ils se sentent brimés. Ils sentent qu'ils n'ont pas leur place comme pères, comme si on leur disait : tu ne peux pas être un bon père. Puis aussi tout le mouvement féministe qui ne croit pas nécessairement que l'homme peut changer puis tout ça, tout ce poids-là fait qu'on oublie souvent tout leur potentiel de père puis d'humain.

En résumé, les intervenants ont évoqué des défis auxquels ils font face et qui limitent leurs interventions sur la paternité. Bien que certains défis mentionnés soient d'ordre individuel, rattachés aux pères, d'autres se situent sur le plan organisationnel (composition, durée et mandat des programmes, isolement professionnel, manque d'outils appropriés et de formation), ainsi que sur le plan social (les lois et politiques sociales sur la protection des enfants, la socialisation masculine, les normes culturelles et les représentations de la VC).

\section{DISCUSSION}

Dans le cadre de cette étude qualitative, nous avons effectué des groupes de discussion et des entrevues semi-dirigées avec des intervenants qui animent des groupes d'aide pour hommes aux comportements violents, afin de comprendre comment ils interviennent sur la paternité des hommes, de même que les enjeux qu'ils rencontrent dans ce domaine. Puisque cette recherche n'a été réalisée que dans trois organismes, deux au Nouveau-Brunswick et un au Québec, les résultats ne peuvent être généralisés aux autres organismes de ces provinces, mais ils pourraient être transférables à d'autres organismes traitant de la VC dans lesquels le contexte organisationnel et social seraient semblables. Cette étude cherchait à comprendre en profondeur la façon dont certains intervenants prennent en considération la paternité au cours de leur pratique.

Lors des entrevues, les intervenants ont évoqué qu'ils visent certains objectifs qui touchent la paternité des hommes, à savoir : la responsabilisation des comportements violents et de leurs effets sur leur enfant ; la co-construction d'une identité de père plus positive ; la valorisation du rôle du père et l'éducation concernant les besoins des enfants et les façons positives d'exercer la discipline. II est intéressant de constater qu'ils misent sur des objectifs en lien avec les forces et les compétences des pères, ce qui est en lien avec une orientation nouvelle et plus large en intervention sociale qui s'éloigne des approches «punitives ». Les intervenants utilisent non seulement des approches visant à confronter, à resocialiser et à éduquer, mais s'inspirent aussi des approches postmodernes, telles que l'approche centrée sur les solutions et l'approche narrative, soit des approches qui misent sur les forces les diverses facettes de l'identité des hommes (Lehmann et Simmons, 2009 ; White, 1991). Ces approches ont été très récemment 
incluses dans les interventions auprès des hommes aux comportements violents (AugustaScott, 2009).

Par ailleurs, les participants à notre étude ont déploré que le mandat des programmes qu'ils offrent se limite à intervenir sur la violence, ce qui exige que certains thèmes soient abordés en premier. Donc, ils manquent de temps pour aborder en profondeur la paternité. De tels défis ont également été identifiés dans l'étude d'Edin et al. (2009) menée auprès d'intervenants suédois qui accompagnent des hommes aux comportements violents. De plus, étant donné qu'il n'y a pas de groupe spécifique pour les pères, les intervenants nous ont dit qu'ils hésitaient parfois à aborder des thèmes touchant la paternité par crainte de perdre l'intérêt des hommes du groupe qui ne sont pas des pères. II semble donc que ce soit le nombre de pères dans le groupe ou le désir des hommes de parler de leur paternité qui déterminent le temps consacré à la paternité.

Les programmes Fathering After Violence et Caring Dads ont été constitués spécifiquement pour scruter à la fois la violence et la paternité. En plus des objectifs mentionnés ci-dessus par les intervenants, ces programmes visent à améliorer les habiletés parentales, à développer des pratiques paternelles qui tiennent compte du développement de l'enfant et à favoriser un engagement paternel plus soutenu (Areán et Davis, 2007 ; Peled et Perel, 2007 ; Scott et al., 2007). Également, ces auteurs considèrent important d'aborder les attitudes des pères à propos de la relation entre la mère et les enfants, de même que l'interaction du père avec la mère (Scott et al., 2007). Comme l'indiquent certaines recherches, les hommes aux comportements violents sont susceptibles de minimiser les capacités parentales de la mère, de nuire à la relation entre l'enfant et la mère et d'utiliser les périodes d'échange des enfants et de visites pour insulter et dénigrer la mère, encourager l'enfant à être contre la mère et contrôler la mère (Bancroft et al., 2012 ; Jaffe et al., 2003). Ainsi, ces aspects doivent être considérés en intervention auprès des hommes. Scott et al. (2007) indiquent également que, dans les cas où la mère n'est pas en mesure d'affronter certaines responsabilités parentales en raison de problèmes de santé mentale ou d'abus de substance, les intervenants doivent reconnaître ces enjeux pour les pères et encourager ceux-ci à s'assurer que les besoins de leurs enfants soient comblés. Les intervenants doivent toutefois miser sur le fait qu'aucun membre de la famille ne mérite d'être l'objet de violence.

Bien que les intervenants de notre recherche aient précisé qu'ils dirigent les pères vers des ressources particulières pour ce qui est du développement de leurs habiletés parentales, certains auteurs sont prudents sur ce point. Scott et al. (2007) signalent que le développement des habiletés parentales des hommes ayant des comportements violents doit se faire dans un contexte où la dynamique de violence et la sécurité de la mère et de l'enfant sont prises en compte, ce qui n'est pas le cas des programmes visant le développement des compétences parentales.

Offering parenting intervention to violent men prior to a successful completion of intervention directed at the violent behavior could strengthen denial of the violence by seeming to reframe their problem as that of deficient parenting (Perel et Peled, $2008: 478$ ). 
Les intervenants de notre étude regrettent l'absence d'outils d'intervention et de formations pour intervenir sur la paternité, car ils se sentent dépourvus face à certains dilemmes qu'ils rencontrent dans leur pratique. Par exemple, ils ne savent pas comment aborder la paternité des hommes lorsque la socialisation masculine crée un frein à l'ouverture des pères sur les sentiments qu'ils ressentent par rapport à la relation avec leurs enfants. Ce constat trouve un écho dans les travaux d'Edin et al. (2009) qui mentionnent que les intervenants en VC trouvent malaisé d'intégrer le thème de la paternité, puisque les hommes tendent à éviter le sujet. Deslauriers (2008: 23) note que les hommes qui adhèrent aux stéréotypes sexuels peuvent tenter de masquer leur honte et leur souffrance par des attitudes d'agressivité, de dépit et d'indifférence. La socialisation masculine exerce une pression profonde sur les hommes, surtout les plus traditionnalistes, pour qu'ils taisent leur vulnérabilité, celle-ci étant perçue comme une faiblesse (De Montigny et al., 2009).

De la même façon, un défi pour les intervenants dans le domaine de la VC est, d'une part, de reconnaître la vulnérabilité des pères aux prises avec des comportements de violence et d'être attentif à leur souffrance et, d'autre part, de condamner leur violence et protéger les personnes victimes (Featherstone et Peckover, 2007 ; Peled et Perel, 2007). Selon Peled et Perel (2007: 90), une telle dualité dans la pratique est essentielle:

The challenge facing anyone who wishes to intervene with men who batter as fathers is how to do it all-protect and respect, trust and suspect, empathize and criticize. Our answer, in one sentence, is "maintain this duality in practice. This implies a condemnation of the harm the fathers cause to the children and to the children's mother and striving to put a stop to the abuse, while also being attentive to the fathers' distress and providing them with support."

Les hommes aux comportements violents doivent être rendus responsables de leurs actes, mais les diverses sphères de leur vie doivent être prises en considération, afin d'engager avec eux un dialogue plus ouvert et moins opposant (Augusta-Scott, 2009). Comme l'écrit Turcotte (2012), être respectueux et à l'écoute de l'autre ne signifie pas que nous sommes nécessairement d'accord, mais plutôt que nous sommes présents et intéressés à découvrir l'univers subjectif de l'autre. « C'est leur donner raison de s'ouvrir, et de considérer la possibilité de devenir un homme vrai plutôt que de continuer à essayer d'être un vrai homme, comme le prescrit la masculinité dominatrice » (Turcotte, 2012 : 124). Cette posture en tant qu'intervenant est d'autant plus importante dans le cas des hommes «plus » traditionnels pour lesquels l'expression de leur souffrance est vécue comme une humiliation (Kilmartin, 2010). Comme l'explique Augusta-Scott (2009), en ignorant la souffrance et la culpabilité des hommes, les intervenants risquent de reproduire le discours sur la socialisation masculine en laissant supposer que les hommes sont à l'abri de la souffrance et de la peur.

\section{CONCLUSION}

Dans le cadre de cet article, nous avons présenté les résultats d'une étude qualitative portant sur la façon dont les intervenants, animant des groupes pour hommes ayant des comportements violents, abordent la paternité. Ces intervenants nous ont parlé du fait qu'ils ont en tête quelques objectifs qui touchent la paternité, mais ils trouvent difficile d'approfondir cet aspect en raison de certains obstacles auxquels ils font face. Outre les obstacles rattachés aux 
pères, les intervenants déplorent la présence de limites organisationnelles (isolement professionnel, manque d'outils appropriés et de formation) et sociales (socialisation masculine, normes et valeurs culturelles et sociales). Ainsi, les intervenants ne réussissent pas à considérer certains objectifs importants mentionnés dans les écrits portant sur la paternité en contexte de VC. En outre, le fait de diriger les pères qui ont des comportements de VC vers des programmes traditionnels axés sur les habiletés parentales peut être risqué, car ces programmes ne tiennent pas compte de la dynamique de cette violence.

Des avenues de recherche et d'intervention peuvent mieux soutenir les intervenants dans leur pratique. Dans ce sens, les programmes Caring Dads et Fathering After Violence, qui visent particulièrement la paternité des hommes aux comportements violents, offrent des pistes intéressantes, mais ils n'ont pas été traduits en français et adaptés dans des provinces canadiennes autres que l'Ontario. Des recherches futures devraient explorer ces possibilités. La mise en place de ces programmes est une voie particulièrement intéressante car, en plus des bénéfices susmentionnés, ils permettraient de relever d'autres défis, notamment de clarifier le mandat des organismes et des intervenants en ce qui concerne la paternité, et de résoudre les défis liés à la composition des groupes et au temps restreint à consacrer à la paternité.

BOURASSA, Chantal

Professeure agrégée École de service social Université de Moncton

LABARRE, Michel

Étudiant au doctorat École de service social Université Laval

TURCOTTE, Pierre

Professeur agrégé École de service social Université Laval

LESSARD, Geneviève

Professeure titulaire

École de service social Université Laval

LETOURNEAU, Nicole Professeure

Faculté de soins \& Médecine (Pédiatrie) Université de Calgary 


\section{BIBLIOGRAPHIE}

Areán, J.C., et L. Davis (2007). «Working with Fathers in Batterer Intervention Programs: Lessons from the Fathering after Violence Project », dans J.L. Edleson et O.J. Williams (dir.), Parenting by Men who Batter: New Directions for Assessment and Intervention, New York (NY), Oxford University Press, p. 118-130.

Augusta-Scott, T. (2009). «A Narrative Therapy Approach to Conversations with Men about Perpetrating Abuse », dans P. Lehmann et C.A. Simmons (dir.), Strength-Based Batterer Intervention: A New Paradigm in Ending Family Violence, New York (NY), Springher Publishing Company, p. 113-135.

Babcock, J.C., C.E. Green et C. Robie (2004). « Does Batterers' Treatment Work? A MetaAnalytic Review of Domestic Violence Treatment », Clinical Psychology Review, vol. 23, $\mathrm{n}^{\circ} 8$, p. 1023-1053.

Bancroft, L., Silverman, J. et Richie, D. (2012) The Batterer as Parent: Addressing the impact of domestic violence on family dynamics, Los Angeles (CA) : Sage.

Blanc-Sahnoun, P., et B. Dameron (2009). Comprendre et pratiquer l'approche narrative, Paris, InterEditions.

Bourassa, C., P. Turcotte, G. Lessard, M. Labarre (2013). « La paternité en contexte de violence conjugale », Revue internationale de l'éducation familiale, vol. 1, nº 33, p. 149-167.

Cabrera, N., et H.E. Peters (2000). « Public policies and father involvement », Marriage and Family Review, vol. 29, $\mathrm{n}^{\circ}$ 4, p. 295-314.

Cronholm, P.F., W. Ellison, S. Mazzella, M. Witherspoon, J.B. Lim, T. Lapp et G. Coleman (2011). "Fathering Agency Staff Members' Perspectives on Domestic Violence Perpetration », American Journal of Men's Health, vol. 5, n 1, p. 47-56.

De Montigny, F., A. Devault, C. Lacharité, A. Quéniart, D. Dubeau, J.-M. Miron, A. Fleurant, C. Gervais, J.-F. Dragon, D. Este, N. St-Amand, J.-M. Bélanger, F. Lozier (2009). «L'enseignement des enjeux de la paternité dans les universités canadiennes», Reflets : revue d'intervention sociale et communautaire, vol. 15, $\mathrm{n}^{\circ} 1$, p. 102-119.

De Shazer, S. (1999). Clés et solutions en thérapie brève, Bruxelles, Satas,

Deslauriers, J.-M. (2008). « Paternités exclues et la formation en travail social », Les Politiques sociales, $\mathrm{n}^{\circ} 1-2$, p. 83-98.

Edin, K. E., Högberg, U., Dahlgren, L. et A. Lalos (2009). « The Pregnancy put The Screws on: Discourses of Professionals Working with Men Inclined to Violence », Men and Masculinities, vol, 11, n 3 , p. 307-324.

Edleson, J.L., et O.J. Williams (2007). Parenting by Men who Batter Women: New Directions for Assessment and Intervention, New York (NY), Oxford University Press.

Featherstone, B., et S. Peckover (2007). «Letting them get away with it: Fathers, domestic violence and child welfare », Critical Social Policy, vol. 27, $n^{\circ} 2$, p. 181-202. 
Fleck-Henderson, A., et J.C. Areán (2004). Fathering after violence: Curriculum guidelines and tools for batterer intervention programs. San Francisco (CA), Family Violence Prevention.

Guille, L. (2004). « Men Who Batter And Their Children: An Integrated Review », Aggression and Violent Behavior, vol. 9, n 2, p. 129-163.

Harne, J. (2011). Violent Fathering and The Risks to Children, Pristol, The Policy Press.

Holden, G.W., et T. Barker (2004). « Fathers in Violent Homes », dans M.E. Lamb (dir.), The Role of the Father in Child Development, Hoboken (NJ), Wiley, p. 417-445.

Huberman, M.A., et B.M. Miles (1991). Analyse des données qualitatives : recueil de nouvelles méthodes, Bruxelles, De Boeck-Wesmael.

Jaffe, P., C.V. Crooks et D.A. Wolfe (2003). « Legal Policy and Response to Children Exposed to Domestic Violence: The Need to Evaluate Intended and Unintended Consequences », Clinical Child and Family Psychology Review, vol. 6, n 3, p. 205-213.

Kilmartin, C. (2010). The Masculine Self, Cornwall-on-Hudson (NY), Sloan Publishing.

Lehmann, P., et C.A. Simmons (2009), Strength-Based Batterer Intervention, New York (NY), Springher Publishing Company

Lecomte, C., et A. Richard (1999). «La psychothérapie humaniste-existentielle d'hier à demain », Revue québécoise de Psychologie, vol. 20, n² 2, p. 189-205.

Lessard, G., Flynn, C., Turcotte, P., Damant, D., Vézina, J.- F., Godin, M.- F., Paradis, F., Delisle, R., Alcedo, Y., Juneau, L., Rock, L., et Rondeau-Cantin, S. (2010). "Child custody issues and co-occurrence of intimate partner violence and child maltreatment: controversies and points of agreement amongst practitioners». Child \& Family Social Work, vol. 12, n 4, p. 492-500.

Macdonald, H., M. McKenzie et E. Fisch (2009). « Bad Mothers and Invisible Fathers' Parenting in the Context of Domestic Violence », Discussion Paper, $\mathrm{n}^{\circ} 7$, Domestic Violence Resource Centre, Victoria (BC).

Mandel, D. (2002). « Working with Batterers as Parents: What Would a Curriculum Look Like? », Issues in Family Violence, vol. 4, $\mathrm{n}^{\circ} 3$.

Mayer, R. (2001). « Le constructivisme et les problèmes sociaux », dans H. Dorvil et R. Mayer (dir.), Problèmes sociaux, tome I: Théories et méthodologies, Sainte-Foy (QC), Presses de l'Université du Québec.

Mukamurera, J., J. Lacourse, Y. Couturier (2006). « Des avancées en analyse qualitative : pour une transparence et une systématisation des pratiques », Recherches qualitatives, vol. 26, $\mathrm{n}^{0} 1$, p. $110-138$.

Neugut, T.B., J.L. Edleson et R.M. Tolman (2010). «Children Exposed to Domestic Violence: Prevention Approaches with Mothers and Fathers », dans S. Alexander, R. Alexander et N. Guterman (dir.), Prevention of Child Maltreatment, St. Louis (MO), G.W. Medical Publishing. 
Paillé, P., et A. Mucchielli (2012). L'analyse qualitative en sciences humaines et sociales, $3^{\mathrm{e}}$ édition, Paris, Armand Collin.

Peled, E., et G. Perel (2007). «A Conceptual Framework for Fathering Intervention with Men who Batter », dans J. L. Edleson et O. J. Williams (dir.), Parenting by Men who Batter: New Directions for Assessment and Intervention, New York (NY), Oxford University Press, p. 85101.

Perel, G., et E. Peled (2008). "The Fathering of Violent Men Constriction and Yearning », Violence Against Women, vol. 14, n 4, p. 457-482.

Roulet, M. (2003). Fatherhood Programs and Domestic Violence: A Technical Assistance Report, Milwauke, Center on Fathers, Families, and Public Policy.

Salisbury, E.J, K. Henning et R. Holdford (2009). « Fathering by Partner-Abusive Men: Attitudes on Children's Exposure to Interparental Conflicts and Risk Factors for Child Abuse », Child Maltreatment, vol. 14, $\mathrm{n}^{\circ} 3$, p. 232-242.

Scott, K.L., K.J. Francis, C.V. Crooks, M. Paddon et D.A. Wolfe (2007). " Guidelines for Intervention with Abusive Fathers », dans J.L. Edleson et O.J. Williams (dir.), Parenting by Men who Batter: New Directions for Assessment and Intervention, New York (NY), Oxford University Press, p. 102-117.

Scott, K.L., et C.V. Crooks (2006). «Preliminary Evaluation of an Intervention Program for Maltreating Fathers », Brief Treatment and Crisis Intervention, vol. 7, n 3, p. 224-238.

Scott, K.L., et V. Lishak (2012). « Intervention For Maltreating Fathers: Statistically And Clinically Significant Change », Child Abuse \& Neglect, vol. 36, n 9, p. 680-684.

Schoppe-Sullivan, S.J., S.C. Mangelsdorf, C.A. Frosch et J.L. McHale (2004). « Associations Between Coparenting and Marital Behavior from Infancy to the Preschool Years », Journal of Family Psychology, vol. 18, nº 1, p. 194-207.

Stover, C.S., T.J. McMahon et C. Easton (2011). « The Impact of Fatherhood on Treatment Response for Men with Co-Occuring Alcohol Dependence and Intimate Partner Violence », American Journal Drug Alcohol Abuse, vol. 37, $\mathrm{n}^{\circ}$ 1, p. 74-78.

Stover, C.S. (2013). «Fathers for Change: A new approach to working with fathers who perpetrate intimate partner violence », Journal of the American Academy of Psychiatry and the Law, vol. 41, $\mathrm{n}^{\circ}$ 1, p. 65-71.

Turcotte, P. (2012). Sortir la violence de sa vie. Itinéraires d'hommes en changement, Québec (QC), Presses de l'Université Laval.

Turcotte, P. (1997). « Intervention de groupe auprès des conjoints violents et logique du contrôle social », Service social, vol. 46, $n^{\text {os }} 2-3$, p. 227-239.

White, M. (1991). « Deconstruction and Therapy », Dulwich Center Newsletter, vol. 3, p. 21-40.

Williams, O.J., J.L. Boggess et J. Carter (2001). « Fatherhood and Domestic Violence: Exploring the Role of Men who Batter in the Lives of their Children », dans S.A. Graham-Bermann et J.L. Edleson (dir.), Domestic Violence in the Lives of Children: The Future of Research, 
Intervention, and Social Policy, Washington (DC), American Psychological Association, p.157-188. 